\title{
A comparison of three rapid methods for the detection of $\beta$-lactamase activity in Haemophilus influenzae
}

\author{
A. SKINNER AND R. WISE \\ From the Department of Medical Microbiology, Dudley Road Hospital, Birmingham B18 7QH, UK
}

SUMMARY Three methods for rapidly detecting $\beta$-lactamase activity in Haemophilus influenzae are compared. The chromogenic cephalosporin method was found to be the most easily performed and the reagents could be stored for up to three weeks. The phenol red method was simple to perform but the iodometric method was more time consuming. All three tests gave identical results.

During the last few years there have been many reports of clinical isolates of Haemophilus influenzae resistant to ampicillin (Williams and Cavanagh, 1974; Thomas et al., 1974). This resistance is due to an $\mathbf{R}$ factor (Tem) mediated $\beta$-lactamase production in these strains (Mederios and O'Brien, 1975). The usual methods of demonstrating ampicillin resistance are by routine sensitivity testing of the isolate. Laboratories using well controlled techniques usually experience little difficulty in identifying such strains, although this will result in a delay of about one day. A simple method of screening $\boldsymbol{H}$. influenzae isolates has obvious attractions, especially in testing strains from the cerebrospinal fluid.

Three methods were compared in this study. The phenol red method as described by Escamilla (1976) detects a decrease in $\mathrm{pH}$ when the $\beta$-lactamase hydrolyses benzylpenicillin to penicilloic acid. Iodometric detection of $\beta$-lactamase production has been widely used to test for this enzyme in many bacteria (Perret, 1954; Cooper et al., 1966), including $H$. influenzae (Catlin, 1975). The novel chromogenic cephalosporin, $87 / 312$, described by O'Callaghan et al. (1972) is readily hydrolysed by the Tem $\beta$ lactamase and the products of this hydrolysis are coloured a brick red.

\section{Material and methods}

Twenty-four strains of $H$. influenzae isolated from clinical material were collected which were known to show a range in sensitivity to ampicillin. The minimum inhibitory concentration (MIC) of ampicillin

Received for publication 19 April 1977 was determined by an agar plate dilution method. Levinthals agar was prepared from Oxoid Isosensitest agar to which $10 \%$ human blood had been added. Ampicillin was added to the agar to give final concentrations of 0.125 to $16 \mu \mathrm{g} / \mathrm{ml}$ and the plates were inoculated using a Denley multipoint inocu 8 lator. The final inoculum was approximately $10^{2}$ to $10^{3}$ colony-forming units per spot. The plates were incubated at $37^{\circ} \mathrm{C}$ in $10 \% \mathrm{CO}_{2}$. After 18 hours incubation the MIC was defined as the lowest concentration of ampicillin which failed to inhibit the growth of $99 \%$ of the original inoculum.

PHENOL RED METHOD

The test reagent was prepared by reconstituting a vial of 1000000 units of benzylpenicillin with $4.5 \mathrm{ml}$ of distilled water and then adding $0.5 \mathrm{ml}$ of $0.5 \%$ aqueous solution of phenol red. One drop of $1.0 \mathrm{M} \mathrm{NaOH}$ was then added to cause a violet colour change in the solution. Colonies from an overnight culture of the test organisms on chocolate agar were suspended in $0.5 \mathrm{ml}$ normal saline in a well of a plastic WHO plate to give an opacity approximately similar to that of a No. 5 MacFarland nephelometer standard. A colour change to yellow, which should occur within one minute, was noted.

\section{CHROMOGENIC CEPHALOSPORIN METHOD}

The method is a modification of that described by Kammer et al. (1975). A $0.5 \mathrm{mg} / \mathrm{ml}$ solution of $87 / 312^{1}$ was made up in $0.05 \mathrm{M}$ potassium phosphate buffer, $\mathrm{pH} \mathrm{7 \cdot 0.}$. The strains to be tested were suspended in normal saline as for the phenol red method in wells and $50 \mu \mathrm{l}$ of the cephalosporin Laboratories, Greenford, Middlesex UB6 0HE. 
reagent was added and a colour change to red noted over the next one to two minutes.

\section{IODOMETRIC METHOD}

A starch indicator was prepared by dissolving $1.0 \mathrm{~g}$ of soluble starch in $100 \mathrm{ml}$ of boiling water. The iodine reagent consisted of $2.03 \mathrm{~g}$ of iodine and $53.2 \mathrm{~g}$ of potassium iodide dissolved in $100 \mathrm{ml}$ of distilled water and then stored in a brown bottle.

A solution of 10000 units of benzylpenicillin per $\mathrm{ml}$ was prepared in phosphate buffer $\mathrm{pH} 7 \cdot 0$. The test was conducted by adding to a well $0.5 \mathrm{ml}$ of the penicillin solution. A similar inoculum, as described above, of the organisms was added. The plates were left at room temperature, approximately $21^{\circ} \mathrm{C}$, for two hours and then 2 drops of the starch solution were added followed by 1 drop of iodine reagent. A colour change from dark blue to colourless was noted over the next 10 minutes.

In all three methods a $\mathrm{Tem}^{+}$and $\mathrm{Tem}^{-}$Escherichia coli control was included. All three methods were repeated using the original reagents stored at $4^{\circ} \mathrm{C}$ at intervals of up to three weeks.

\section{Results}

In the Table are shown the sensitivities to ampicillin of the 24 strains of $\boldsymbol{H}$. influenzae. Nine strains could be said to be definitely resistant to ampicillin with MICs of greater than $1 \mu \mathrm{g} / \mathrm{ml}$. The remainder were sensitive.

Table Comparison of three methods of detecting $\beta$-lactamase production in $\mathrm{H}$. influenzae

\begin{tabular}{|c|c|c|c|c|}
\hline Strain no. & $M I C(m g / l)$ & Phenol red & Iodometric & $87 / 312$ \\
\hline 1 & 0.25 & - & - & - \\
\hline 2 & 0.25 & - & - & - \\
\hline 3 & 1.0 & - & - & - \\
\hline 4 & $>16$ & + & + & + \\
\hline 5 & 0.5 & - & - & - \\
\hline 6 & 0.5 & - & - & - \\
\hline 7 & 0.5 & - & - & - \\
\hline 8 & 0.25 & - & - & - \\
\hline 9 & 0.25 & - & - & - \\
\hline 10 & 8 & + & + & + \\
\hline 11 & 0.25 & - & - & - \\
\hline 12 & 0.25 & - & - & - \\
\hline 13 & 1.0 & - & - & - \\
\hline 14 & 4 & + & + & + \\
\hline 15 & 4 & + & + & + \\
\hline 16 & 0.25 & - & - & - \\
\hline 17 & 0.25 & - & - & - \\
\hline 18 & 8 & + & + & + \\
\hline 19 & 8 & + & + & + \\
\hline 20 & 8 & + & + & + \\
\hline 21 & 0.25 & - & - & - \\
\hline 22 & 1 & - & - & - \\
\hline 23 & 8 & + & + & + \\
\hline 24 & 8 & + & + & + \\
\hline
\end{tabular}

colour change indicative of $\beta$-lactamase activity.
All three methods gave identical results detecting those strains which had MICs of greater than 1 $\mu \mathrm{g} / \mathrm{ml}$. Differences between the methods were of a technical nature, the chromogenic cephalosporin method being the simplest and most rapid to perform, taking only one to two minutes and the reagents could still be used after three weeks' storage at $4^{\circ} \mathrm{C}$. The phenol-red method was also technically very simple but fresh reagents had to be made daily. The iodometric method took over two hours to perform and also had the disadvantage that the starch solution had to be freshly prepared on each occasion.

\section{Discussion}

Concern as to the possibility of an isolate of $H$. influenzae being resistant to ampicillin has led to the advice on the treatment of Haemophilus meningitis being changed (American Academy of Pediatrics Committee on Infectious Diseases, 1975). It would be an advantage to the clinician if he could be informed rapidly as to the likely sensitivity to ampicillin of an isolate from, in particular, cerebrospinal fluid. The three tests described are all simple to perform and, except with the iodometric method, an answer can be obtained within a matter of minutes. In our opinion, the use of $87 / 312$ was the most easily performed method.

The routine sensitivity testing of isolates of $H$. influenzae is not without problems. Variations in media, inoculum size, and disc strength (Annotation, 1976) make these organisms among the more difficult to test by the disc diffusion method. It is not suggested that one of the rapid methods described should supplant the established procedures, but a rapid screening test before disc testing would be a useful addition to laboratory practice. The method can also be used to detect or confirm the presence of $\beta$-lactamase producing Neisseria gonorrhoeae by simply suspending an overnight culture of these organisms in place of the $H$. influenzae.

\section{References}

American Academy of Pediatrics Committee on Infectious Diseases (1975). Ampicillin-resistant strains of Hemophilus influenzae type B. Pediatrics, 55, 145-146.

Annotation (1976). Haemophilus influenzae. Lancet, 2 , 776-777.

Catlin, B. W. (1975). Iodometric detection of Haemophilus influenzae $\beta$-lactamase: rapid presumptive test for ampicillin resistance. Antimicrobial Agents and Chemotherapy, 7, 265-270.

Cooper, R. G., Brown, G. W., and Vesey, B. V. (1966). Iodometric detection of staphylococcal penicillinase in clinical microbiology. Australian Journal of Experi- 
mental Biology and Medical Science, 44, 715-717.

Escamilla, J. (1976). Susceptibility of Haemophilus influenzae to ampicillin as determined by use of a modified, one-minute beta-lactamase test. Antimicrobial Agents and Chemotherapy, 9, 196-198.

Kammer, R. B., Preston, D. A., Turner, J. R., and Hawley, L. C. (1975). Rapid detection of ampicillinresistant Haemophilus influenzae and their susceptibility to sixteen antibiotics. Antimicrobial Agents and Chemotherapy, 8, 91-94.

Mederios, A. A., and O'Brien, T. F. (1975). Ampicillinresistant Haemophilus influenzae type B. Läncet, 1, 716719.
O'Callaghan, C. H., Morris, A., Kirby, S. M., and Shingler, A. H. (1972). Novel method for detection of $\beta$ lactamases by using a chromogenic cephalosporin substrate. Antimicrobial Agents and Chemotherapy, 1, 283-288.

Perret, C. J. (1954). Iodometric assay of penicillinase. Nature (London), 174, 1012-1013.

Thomas, W. J., McReynolds, J. W., Mock, C. R., and Bailey, D. W. (1974). Ampicillin-resistant Haemophilus influenzae meningitis (Letter). Lancet, 1, 313.

Williams, J. D., and Cavanagh, P. (1974). Ampicillin resistant Haemophilus influenzae meningitis (Letter). Lancet, 1, 864. 